\title{
Doença pulmonar obstrutiva crônica: análise da deglutição em pacientes hospitalizados
}

\author{
Chronic obstructive pulmonary disease: \\ swallowing analysis in hospitalized patients
}

\section{Enfermedad pulmonar obstructiva crónica: análisis de la deglución en pacientes hospitalizados}

\author{
Flávia Caroline Fontoura Aguiar* \\ Simone Lourenço Vale* \\ Laélia Cristina Caseiro Vicente**
}

\section{Resumo}

Objetivos: investigar a ocorrência de disfagia e o risco de broncoaspiração em pacientes hospitalizados com doença pulmonar obstrutiva crônica, e verificar se há associação com as características sociodemográficas, clínicas, do sistema estomatognático e da dinâmica de deglutição. Métodos: estudo descritivo observacional e retrospectivo com coleta de dados no prontuário eletrônico. Foram avaliados 27 prontuários de pacientes com doença pulmonar obstrutiva crônica, internados em um hospital público de Minas Gerais no período de julho de 2012 a julho de 2015 e com avaliação de deglutição. Para verificar a presença de disfagia e o risco de broncoaspiração foi utilizado o protocolo The Mann Assessment of Swallowing Ability. Foram consideradas associações estatisticamente significantes aquelas cujo p-valor foi $\leq 0,05$ e intervalo de confiança de $95 \%$. Resultados: Dos 27 pacientes avaliados pela equipe de fonoaudiologia, $41,7 \%$ tinham disfagia, sendo a gravidade leve mais prevalente (82\%) e 19\% tinham risco de broncoaspiração. Não houve predomínio de alguma fase da deglutição alterada. Houve associação entre presença de disfagia e a cooperação do paciente, e o risco de aspiração com o tempo

\footnotetext{
* Hospital Risoleta Tolentino Neves/ UFMG, Belo Horizonte, MG, Brasil.

**Universidade Federal de Minas Gerais, Belo Horizonte, MG, Brasil.

Contribuição dos autores:

FCFA: responsável pela concepção e delineamento do projeto de pesquisa, coleta e análise dos dados e elaboração do manuscrito; SLV: responsável pela concepção e delineamento do projeto de pesquisa, análise dos dados; LCCV: responsável pela concepção e delineamento do projeto de pesquisa, análise dos dados e elaboração do manuscrito.
}

E-mail para correspondência: Flavia Caroline Fontoura Aguiar - flaviafono.aguiar@gmail.com Recebido: 22/03/2017

Aprovado: 27/02/2018 
de trânsito oral. Conclusão: Os resultados mostraram relação entre disfagia e risco de aspiração em pacientes internados com doença pulmonar obstrutiva crônica, sugerindo a necessidade de investigação da dinâmica da deglutição durante a hospitalização.

Palavras-chave: Transtornos de deglutição; Doença Pulmonar Obstrutiva Crônica; Disfagia.

\section{Abstract}

Objective: To investigate the occurrence of dysphagia and the risk of bronchoaspiration in hospitalized patients with chronic obstructive pulmonary disease and to verify if there is any association between the severity of dysphagia and the risk of aspiration with sociodemographic aspects, clinical characteristics, stomatognathic system and swallowing dynamics. Methods: descriptive observational study using the retrospective type of electronic data collection. We evaluated 27 medical records of patients with chronic obstructive pulmonary disease admitted to a public hospital in Minas Gerais in the period from July 2012 to July 2015 and with swallowing assessment. To verify the presence of dysphagia and the risk of bronchoaspiration the protocol The Mann Assessment of Swallowing Ability was used. Statistically significant associations were those whose p-value was $\leq 0.05$ and $95 \%$ confidence interval. Results: Between the 27 patients evaluated by the speech-language pathology team, $41.7 \%$ had dysphagia, with light severity being the majority $(82 \%)$ and $19 \%$ had a risk of bronchoaspiration. There was no predominance of any phase of altered swallowing. There was an association between the presence of dysphagia and patient cooperation, and the risk of aspiration with oral transit time. Conclusion: The results showed a relationship between dysphagia and aspiration risk in hospitalized patients with chronic obstructive pulmonary disease, suggesting the need to investigate the dynamics of swallowing during hospitalization.

Keywords: Deglutition disorders; Chronic obstructive pulmonary disease; Dysphagia.

\section{Resumen}

Objetivos: investigar la ocurrencia de disfagia y el riesgo de broncoaspiración en pacientes hospitalizados con enfermedad pulmonar obstructiva crónica y verificar si hay asociación con las características sociodemográficas, clínicas, del sistema estomatognático y la dinámica de la deglución. Métodos: Estudio observacional descriptivo de tipo retrospectivo con recogida de datos en registros médicos electrónicos. Se evaluaron 27 registros médicos de pacientes con enfermedad pulmonar obstructiva crónica, internados en un hospital público de Minas Gerais en el período de julio de 2012 a julio de 2015 y con evaluación de deglución. Para verificar la presencia de disfagia y el riesgo de broncoaspiración se utilizó el protocolo The Mann Assessment of Swallowing Ability. Se consideraron asociaciones estadísticamente significativas aquellas cuyo valor de $\mathrm{p}$ fue $\leq 0,05$ e intervalo de confianza de 95\%. Resultados: De los 27 pacientes evaluados por el equipo de fonoaudiología, el 41,7\% tenían disfagia, siendo la gravedad leve más prevalente $(82 \%)$ y el $19 \%$ tenían riesgo de broncoaspiración. No hubo predominio de cualquier fase de la deglución alterada. Hubo asociación entre la presencia de disfagia y la cooperación del paciente, y el riesgo de aspiración con el tiempo de tránsito oral. Conclusión: Los resultados mostraron relación entre disfagia y riesgo de aspiración en los pacientes hospitalizados con enfermedad pulmonar obstructiva crónica, lo que sugiere una necesidad de investigación sobre la dinámica de la deglución durante una hospitalización.

Palabras claves: Trastornos de la deglución; Disfagia; Enfermedad pulmonar obstructiva crónica. 


\section{Introdução}

A doença pulmonar obstrutiva crônica (DPOC) é caracterizada por limitação do fluxo aéreo, que leva a alterações patológicas nos pulmões. A limitação do fluxo aéreo está associada a respostas inflamatórias anormais dos pulmões a partículas ou gases nocivos ${ }^{1}$.

Alguns dos sintomas apresentados na DPOC são: sibilos, tosse produtiva e dispneia ao esforço respiratório, sendo esta, o sintoma associado ao pior prognóstico, com menor capacidade e maior perda da função pulmonar ${ }^{2}$. Além do tabagismo, a predisposição genética, fatores sócio econômicos, exposição à poluição e a partículas pesadas no ar estão relacionados com o aparecimento da $\mathrm{DPOC}^{3}$.

Estudos mostram que a DPOC é a quarta principal causa de morte nos Estados Unidos e na Europa, sendo que o impacto da doença tende a aumentar, devido o envelhecimento da população ${ }^{4}$. No Brasil é a terceira principal causa de morte, tendo apresentado um aumento de $12 \%$ entre 2005 e 2010, o que significa que há aproximadamente 40 mil mortes por ano causadas por DPOC ${ }^{5}$. Estima-se que cinco a seis milhões de brasileiros têm DPOC, sendo que os pacientes tendem a ser concentrados principalmente nas faixas etárias acima de 50 anos $^{6}$.

O diagnóstico de DPOC deve ser considerado em qualquer indivíduo que apresente sintomas característicos e história de exposição aos fatores de risco para a doença, especialmente à fumaça de cigarro ${ }^{7}$

As alterações no padrão respiratório podem influenciar a coordenação entre deglutição e respiração dos indivíduos com $\mathrm{DPOC}^{8}$. Os pacientes acometidos pela doença são mais suscetíveis às alterações deste sincronismo, o que aumenta os riscos de infecções respiratórias e broncoaspirações ${ }^{9}$.

A redução na sensibilidade laringofaríngea pode comprometer o disparo da deglutição, ${ }^{10}$ sendo um fator de risco para a exacerbação da doença $a^{9,10}$. Apesar de a disfagia ser parte dos sintomas apresentados pelos pacientes com DPOC, pouca ênfase é dada para a identificação precoce das alterações de deglutição ${ }^{11}$

A reabilitação fonoaudiológica nas disfagias orofaríngeas visa proteção de vias aéreas inferiores e nutrição do paciente ${ }^{12}$. A atuação do Fonoaudiólogo na equipe multidisciplinar contribui para a redução do tempo de permanência hospitalar e de readmissão hospitalar devido a complicações refe- rentes às alterações das funções motoras orais ${ }^{13}$. A eficácia da fonoterapia é definida com a retomada da alimentação por via oral com valor nutricional e segurança na deglutição ${ }^{14}$.

A disfagia é uma alteração que pode comprometer as condições clínicas e de qualidade de vida do paciente e suas complicações, como desnutrição, desidratação e pneumonia aspirativa (PNM), que podem agravar a recuperação. Assim, em indivíduos com DPOC, as condições respiratórias podem interferir na dinâmica da deglutição e torná-los mais predispostos às complicações da disfagia.

Assim, este estudo visa investigar a ocorrência de disfagia e o risco de broncoaspiração em pacientes com DPOC hospitalizados e verificar se há associação com as características sociodemográficas, clínicas, do sistema estomatognático e da dinâmica de deglutição.

\section{Método}

Trata-se de um estudo descritivo analítico retrospectivo, de amostra não probabilística, de coleta de dados do prontuário eletrônico, de pacientes internados em um hospital público de Belo Horizonte-MG. Trabalho aprovado pelo Comitê de Ética da Universidade Federal de Minas Gerais CAAE 53353616.8.0000.5149 e pelo Núcleo de Ensino, Pesquisa e Extensão do Hospital Risoleta Tolentino Neves $-n^{\circ} 42 / 2015$.

Os critérios de inclusão adotados neste estudo foram pacientes internados no período de julho de 2012 a julho de 2015, com idade superior a 18 anos, com doença pulmonar obstrutiva crônica, avaliados pela equipe de Fonoaudiologia do hospital. Foram excluídos os prontuários de pacientes com doenças neurológicas associadas, psiquiátricas, pacientes sob ventilação mecânica ou com traqueostomia, disfagia esofágica, cirurgias ou neoplasias pulmonares e de cabeça e pescoço, pacientes que faleceram antes da avaliação fonoaudiológica.

Para identificação dos possíveis participantes, o setor de informática do hospital realizou o levantamento dos pacientes com diagnóstico médico de DPOC por meio da Classificação Internacional de Doenças (CID) no sistema de registro informatizado no período estipulado. A partir desse levantamento, 40 pacientes foram identificados e os prontuários eletrônicos foram consultados para verificar quais pacientes se enquadravam aos critérios de inclusão e tinham sido submetidos à 
avaliação fonoaudiológica. Desses, 29 pacientes eram candidatos, todavia dois destes foram excluídos, por apresentarem dados incompletos da avaliação fonoaudiológica.

O diagnóstico de DPOC no hospital onde foi realizado o estudo é feito por um médico clínico geral ou pneumologista, que esteja acompanhando o enfermo, por meio do histórico e exame clínico, espirometria e radiografia do tórax.

Para verificar a presença de disfagia e o risco de broncoaspiração, foi utilizado o protocolo The Mann Assessment of Swallowing Ability (MASA), ${ }^{15}$ no qual inclui a avaliação motora e sensorial das estruturas envolvidas no processo de deglutição e investiga clinicamente a dinâmica da deglutição. Embora o instrumento tenha sido validado para população com AVC (acidente vascular cerebral), optou-se por utilizar esse instrumento porque não há qualquer outro validado para pacientes com DPOC e ele fornece informações relativas aos objetivos deste estudo.

Foram retirados do protocolo MASA, devido ausência de dados nos prontuários, os itens: afasia, respiração (ausculta pulmonar), tosse voluntária e dispraxia. O item disartria, foi adaptado para fala, devido à exclusão de pacientes neurológicos. Após exclusão desses itens, foi realizada a adequação da pontuação da escala de gravidade de disfagia e o risco de broncoaspiração. Assim, os escores entre 151 e 170 pontos indicam ausência de disfagia; entre 142 e 150 pontos - disfagia leve; entre 117 e 141 pontos - disfagia moderada e valores abaixo de 117 pontos - disfagia grave. Com relação ao risco de aspiração, escores entre 145 e 170 pontos indicam ausência de risco; entre 127 e 144 pontos - risco leve; menor ou igual a 126 pontos - risco moderado e valores abaixo de 118 pontos - risco grave.

Os dados coletados foram: sexo, idade, presença de comorbidades (PNM, doenças cardíacas, queda/ fratura, metabólicas), via de alimentação antes da avaliação fonoaudiológica, suporte ventilatório, estado de alerta, cooperação, compreensão, fala, voz, dentição, higiene oral, vedamento labial, movimentação de língua, força de língua, coordenação de língua, reflexo de GAG, mobilidade do palato mole, reflexo de tosse, deglutição de saliva, preparação oral, clareamento do bolo, trânsito oral, fase faríngea, resposta faríngea, frequência respiratória, nível funcional de deglutição por meio da Escala Funcional de Alimentação (Functional Oral IntakeScale - FOIS) ${ }^{16}$ após avaliação da Fonoaudio- logia, gravidade da disfagia, risco de aspiração. A escala FOIS é caracterizada por sete níveis: Nivel 1- Nada por via oral; Nível 2- Dependente de via alternativa com mínima via oral de alimento ou líquido; Nível 3- Dependente de via alternativa com consistente via oral de alimento ou líquido; Nível 4- Via oral total de uma consistência; Nível 5- Via oral total com múltiplas consistências, mas com necessidade de preparo especial ou compensações; Nível 6- Via oral total com múltiplas consistências, mas sem necessidade de preparo especial ou compensações, porém, com restrições para alguns alimentos; Nível 7- Via oral sem restrições ${ }^{16}$

Após a coleta dos dados, foram realizadas análises descritivas das variáveis por meio de distribuição de frequência absoluta e relativa das variáveis categóricas e de síntese numérica das variáveis contínuas. Para análises de associação bivariada foram consideradas três variáveis respostas: 1. Presença e ausência de disfagia; 2 . Risco de aspiração (presente e ausente) e 3. Gravidade da disfagia (leve e moderada). As variáveis explicativas foram agrupadas em dois blocos, sendo o primeiro relacionado à caracterização dos pacientes (sexo, idade, comorbidades, via de alimentação antes da avaliação fonoaudiológica, suporte ventilatório, estado de alerta, cooperação compreensão, fala, voz) e o segundo às características do sistema estomatognático e da deglutição (dentição, higiene oral, vedamento labial, movimentação de língua, força de língua, coordenação de língua, reflexo de tosse, deglutição de saliva, preparação oral, clareamento do bolo, trânsito oral, fase faríngea, resposta faríngea, frequência respiratória). Optou-se por realizar a análise de associação entre todas as variáveis explicativas e as três variáveis respostas, ampliando as possibilidades de análises solicitadas.

As variáveis explicativas também foram recodificadas para reduzir as categorias com poucas informações e melhorar as estimativas das análises. As demais variáveis foram mantidas na forma original para a análise bivariada.

Para as variáveis categóricas, foram utilizados os testes qui-quadrado de Pearson ou Exato de Fisher para análise das associações. Para avaliação da associação entre as variáveis respostas e a variável idade (distribuição normal), foi utilizado o teste T. Foram consideradas associações estatisticamente significantes aquelas cujo $p$-valor foi $\leq 0,05$ e intervalo de confiança de $95 \%$. Para todas as análises 
foi utilizado o programa Statistical Package for the Social Sciences (SPSS), versão 21.0.

\section{Resultados}

A maioria dos pacientes era do sexo feminino $(59,3 \%)$, com idade variando de 55 a 97 anos, média de idade de 74,9 anos (DP=10,7). Dentre as comorbidades, houve maior ocorrência de pneumonia e doenças metabólicas (25,9\% cada), seguida de doença renal $(22,2 \%)$, doenças cardíacas $(18,5 \%)$, doenças digestivas e queda com trauma (3,7\% cada). A flutuação do nível de consciência foi de $51,9 \%$ dos pacientes, $63,0 \%$ eram cooperativos e $92,0 \%$ utilizavam suporte respiratório por meio de ventilação não invasiva (cateter nasal ou máscara facial).

A avaliação fonoaudiológica revelou que $70,4 \%$ tinham compreensão auditiva adequada,
$77,8 \%$ sem anormalidade na fala e $51,9 \%$ tinham alterações na voz. Em relação às condições orais, $63,0 \%$ eram edêntulos e $66,7 \%$ tinham higiene oral adequada. Nenhuma anormalidade foi detectada em relação ao reflexo de GAG $(100,0 \%)$ e ao palato mole $(100,0 \%)$; em relação à movimentação, força e coordenação da língua, estas estavam adequadas em $88,9 \%, 85,2 \%$ e $77,8 \%$ dos pacientes, respectivamente. $\mathrm{O}$ reflexo de tosse estava adequado em $96,3 \%$ dos pacientes e a deglutição de saliva sem anormalidades em $85,2 \%$.

Em relação à deglutição, por meio do instrumento MASA, $41,7 \%$ dos pacientes tinham disfagia, sendo a gravidade leve mais prevalente, e $19 \%$ tinham risco de broncoaspiração. Não houve predomínio de alguma fase da deglutição alterada, já a incoordenação na frequência respiratória durante a avaliação da dinâmica da deglutição foi frequente entre os pacientes avaliados conforme apresentado na Tabela 1.

Tabela 1. Caracterização da deglutição nos pacientes hospitalizados com DPOC

\begin{tabular}{cccc}
\hline Características & & $\mathbf{N}(\mathbf{n = 2 7})$ & $\mathbf{\%}$ \\
\hline Disfagia & Ausente & 16 & 59,3 \\
& Presente & 11 & 41,7 \\
\hline Gravidade $(\mathrm{N}=11)$ & Leve & 9 & 82 \\
& Moderada & 2 & 18 \\
\hline Risco aspiração & Ausente & 22 & 81 \\
& Presente & 5 & 19 \\
\hline FOIS após avaliação/fono & 1 & 12 & 44,5 \\
& 5 & 10 & 37 \\
& 6 & 3 & 11,1 \\
\hline Preparação oral & 7 & 2 & 7,4 \\
\hline Clearance de bolo & Adequada & 25,6 \\
& Alterada & 2 & 7,4 \\
\hline Trânsito oral & Adequada & 24 & 88,9 \\
& Alterada & 3 & 11,1 \\
\hline Fase Faríngea & Adequado & 25 & 7,6 \\
\hline Resposta Faríngea & Alterado & 2 & 78 \\
& Adequada & 21 & 22 \\
\hline Frequência respiratória & Alterada & 6 & 89 \\
& Adequada & 24 & 11 \\
\hline & Alterada & 3 & 14,8 \\
\hline
\end{tabular}

Legenda: $\mathrm{N}$ = número de pacientes, FOIS = Escala Funcional de Alimentação 
A análise de associação entre a presença de disfagia e características sociodemográficas e clínicas dos pacientes revelou que a única variável que se associou à presença de disfagia foi a cooperação do paciente, havendo maior proporção de pacientes pouco cooperativos entre os que tinham disfagia, comparados aos pacientes sem disfagia (Tabela 2).

Tabela 2. Associação entre disfagia e características dos pacientes com DPOC $(\mathrm{N}=27)$

\begin{tabular}{|c|c|c|c|c|c|}
\hline \multirow{3}{*}{$\begin{array}{c}\text { Características } \\
\text { Sexo }\end{array}$} & \multicolumn{4}{|c|}{ Disfagia } & \multirow{3}{*}{ Valor-p* } \\
\hline & \multicolumn{2}{|c|}{ Ausente } & \multicolumn{2}{|c|}{ Presente } & \\
\hline & $\mathbf{N}$ & $\%$ & $\mathbf{N}$ & $\%$ & \\
\hline Masculino & 7 & 41,2 & 4 & 40,0 & \\
\hline Feminino & 10 & 58,8 & 6 & 60,0 & 0,637 \\
\hline Total & 17 & 100,0 & 10 & 100,0 & \\
\hline Comorbidades & $\mathbf{N}$ & $\%$ & $\mathbf{N}$ & $\%$ & \\
\hline PNM & 5 & 29,4 & 2 & 20,0 & \\
\hline Doenças cardíacas & 4 & 23,5 & 1 & 10,0 & \\
\hline Outras & 8 & 47,1 & 7 & 70,0 & 0,488 \\
\hline Total & 17 & 100,0 & 10 & 100,0 & \\
\hline Suporte ventilatório & $\mathbf{N}$ & $\%$ & $\mathbf{N}$ & $\%$ & \\
\hline AA (ar ambiente) & 1 & 5,9 & 1 & 10,0 & \\
\hline Suporte ventilatório & 16 & 94,1 & 9 & 90,0 & 0,613 \\
\hline Total & 17 & 100,0 & 10 & 100,0 & \\
\hline Estado de alerta & $\mathbf{N}$ & $\%$ & $\mathbf{N}$ & $\%$ & \\
\hline Adequado & 10 & 58,8 & 3 & 30,0 & \\
\hline Inadequado & 7 & 41,2 & 7 & 70,0 & 0,147 \\
\hline Total & 17 & 100,0 & 10 & 100,0 & \\
\hline Cooperação & $\mathbf{N}$ & $\%$ & $\mathbf{N}$ & $\%$ & \\
\hline Adequado & 14 & 82,4 & 3 & 30,0 & \\
\hline Inadequado & 3 & 17,6 & 7 & 70,0 & 0,010 \\
\hline Total & 17 & 100,0 & 10 & 100,0 & \\
\hline \multicolumn{6}{|l|}{ Idade } \\
\hline Mediana & \multicolumn{2}{|c|}{77,0} & \multicolumn{2}{|c|}{73,5} & \\
\hline Média & \multicolumn{2}{|c|}{76,2} & \multicolumn{2}{|c|}{72,5} & 0,393 \\
\hline Desvio Padrão & \multicolumn{2}{|c|}{10,7} & \multicolumn{2}{|c|}{10,9} & \\
\hline
\end{tabular}

*Qui-quadrado de Pearson ou Exato de Fisher

Nenhuma das variáveis do sistema estomatognático e deglutição foram associadas à presença de disfagia (Tabela 3).
Não houve associação entre o risco de aspiração e as características sociodemográficas e clínicas dos pacientes com DPOC (Tabela 4) 
Tabela 3. Associação entre disfagia e variáveis do sistema estomatognático e deglutição $(N=27)$

\begin{tabular}{|c|c|c|c|c|c|}
\hline \multirow{3}{*}{$\begin{array}{c}\text { Características } \\
\text { Higienização oral }\end{array}$} & \multicolumn{4}{|c|}{ Disfagia } & \multirow{3}{*}{ Valor-p* } \\
\hline & \multicolumn{2}{|c|}{ Ausente } & \multicolumn{2}{|c|}{ Presente } & \\
\hline & $\mathbf{N}$ & $\%$ & $\mathbf{N}$ & $\%$ & \\
\hline Adequada & 12 & 70,6 & 6 & 60,0 & \\
\hline Inadequada & 5 & 29,4 & 4 & 40,0 & 0,439 \\
\hline Total & 17 & 100,0 & 10 & 100,0 & \\
\hline Vedamento Labial & $\mathbf{N}$ & $\%$ & $\mathbf{N}$ & $\%$ & \\
\hline Adequado & 16 & 94,1 & 8 & 80,0 & \\
\hline Inadequado & 1 & 5,9 & 2 & 20,0 & 0,128 \\
\hline Total & 17 & 100,0 & 10 & 100,0 & \\
\hline Movimentação de Língua & $\mathbf{N}$ & $\%$ & $\mathbf{N}$ & $\%$ & \\
\hline Adequada & 16 & 94,1 & 1 & 10,0 & \\
\hline Alterada & 1 & 5,9 & 9 & 90,0 & 0,303 \\
\hline Total & 17 & 100,0 & 10 & 100,0 & \\
\hline Força de Língua & $\mathbf{N}$ & $\%$ & $\mathbf{N}$ & $\%$ & \\
\hline Adequada & 15 & 88,2 & 8 & 80,0 & \\
\hline Alterada & 2 & 11,8 & 2 & 20,0 & 0,477 \\
\hline Total & 17 & 100,0 & 10 & 100,0 & \\
\hline Coordenação de Língua & $\mathbf{N}$ & $\%$ & $\mathbf{N}$ & $\%$ & \\
\hline Adequada & 15 & 88,2 & 6 & 60,0 & \\
\hline Alterada & 2 & 11,8 & 4 & 40,0 & 0,112 \\
\hline Total & 17 & 100,0 & 10 & 100,0 & \\
\hline Reflexo de Tosse & $\mathbf{N}$ & $\%$ & $\mathbf{N}$ & $\%$ & \\
\hline Adequado & 16 & 94,1 & 10 & 100,0 & \\
\hline Alterado & 1 & 5,9 & 0 & 0,0 & 0,630 \\
\hline Total & 17 & 100,0 & 10 & 100,0 & \\
\hline Deglutição Salivar & $\mathbf{N}$ & $\%$ & $\mathbf{N}$ & $\%$ & \\
\hline Adequada & 16 & 94,1 & 7 & 70,0 & \\
\hline Alterada & 1 & 5,9 & 3 & 30,0 & 0,128 \\
\hline Total & 17 & 100,0 & 10 & 100,0 & \\
\hline Preparação Oral & $\mathbf{N}$ & $\%$ & $\mathbf{N}$ & $\%$ & \\
\hline Adequada & 16 & 94,1 & 9 & 90,0 & \\
\hline Alterada & 1 & 5,9 & 1 & 10,0 & 0,613 \\
\hline Total & 17 & 100,0 & 10 & 100,0 & \\
\hline Clareamento do Bolo & $\mathbf{N}$ & $\%$ & $\mathbf{N}$ & $\%$ & \\
\hline Adequado & 16 & 94,1 & 8 & 80,0 & \\
\hline Alterado & 1 & 5,9 & 2 & 20,0 & 0,303 \\
\hline Total & 17 & 100,0 & 10 & 100,0 & \\
\hline Trânsito Oral & $\mathbf{N}$ & $\%$ & $\mathbf{N}$ & $\%$ & \\
\hline Adequado & 17 & 100,0 & 8 & 80,0 & \\
\hline Alterado & 0 & 0,0 & 2 & 20,0 & 0,128 \\
\hline Total & 17 & 100,0 & 10 & 100,0 & \\
\hline Fase Faríngea & $\mathbf{N}$ & $\%$ & $\mathbf{N}$ & $\%$ & \\
\hline Adequada & 15 & 88,2 & 7 & 70,0 & \\
\hline Alterada & 2 & 11,8 & 3 & 30,0 & 0,249 \\
\hline Total & 17 & 100,0 & 10 & 100,0 & \\
\hline Resposta Faríngea & $\mathbf{N}$ & $\%$ & $\mathbf{N}$ & $\%$ & \\
\hline Adequada & 17 & 100,0 & 8 & 80,0 & \\
\hline Alterada & 0 & 0,0 & 2 & 20,0 & 0,128 \\
\hline Total & 17 & 100,0 & 10 & 100,0 & \\
\hline Frequência Respiratória & $\mathbf{N}$ & $\%$ & $\mathbf{N}$ & $\%$ & \\
\hline Adequada & 2 & 11,8 & 2 & 20,0 & \\
\hline Alterada & 15 & 88,2 & 8 & 80,0 & 0,477 \\
\hline Total & 17 & 100,0 & 10 & 100,0 & \\
\hline
\end{tabular}

* Teste Exato de Fisher 
Tabela 4. Associação entre risco de aspiração e características dos pacientes com DPOC (N=27)

\begin{tabular}{|c|c|c|c|c|c|}
\hline \multirow{3}{*}{$\begin{array}{c}\text { Características } \\
\text { Sexo }\end{array}$} & \multicolumn{4}{|c|}{ Aspiração } & \multirow{3}{*}{ Valor-p* } \\
\hline & \multicolumn{2}{|c|}{ Ausente } & \multicolumn{2}{|c|}{ Presente } & \\
\hline & $\mathbf{N}$ & $\%$ & $\mathbf{N}$ & $\%$ & \\
\hline Masculino & 10 & 43,5 & 1 & 25,0 & \multirow{3}{*}{0,455} \\
\hline Feminino & 13 & 56,5 & 3 & 75,0 & \\
\hline Total & 23 & 100,0 & 4 & 100,0 & \\
\hline Comorbidades & $\mathbf{N}$ & $\%$ & $\mathbf{N}$ & $\%$ & \multirow{5}{*}{0,378} \\
\hline PNM & 5 & 21,7 & 2 & 50,0 & \\
\hline Doenças cardíacas & 5 & 21,7 & 0 & 0,0 & \\
\hline Outras & 13 & 56,5 & 2 & 50,0 & \\
\hline Total & 23 & 100,0 & 4 & 100,0 & \\
\hline Suporte ventilatório & $\mathbf{N}$ & $\%$ & $\mathbf{N}$ & $\%$ & \multirow{4}{*}{0,279} \\
\hline AA (ar ambiente) & 1 & 4,3 & 1 & 25,0 & \\
\hline Suporte ventilatório & 22 & 95,7 & 3 & 75,0 & \\
\hline Total & 23 & 100,0 & 4 & 100,0 & \\
\hline Estado de alerta & $\mathbf{N}$ & $\%$ & $\mathbf{N}$ & $\%$ & \multirow{4}{*}{0,327} \\
\hline Adequado & 12 & 52,2 & 1 & 25,0 & \\
\hline Inadequado & 11 & 47,8 & 3 & 75,0 & \\
\hline Total & 23 & 100,0 & 4 & 100,0 & \\
\hline Cooperação & $\mathbf{N}$ & $\%$ & $\mathbf{N}$ & $\%$ & \multirow{4}{*}{0,477} \\
\hline Adequada & 15 & 65,2 & 2 & 50,0 & \\
\hline Inadequada & 8 & 34,8 & 2 & 50,0 & \\
\hline Total & 23 & 100,0 & 4 & 100,0 & \\
\hline \multicolumn{6}{|l|}{ Idade } \\
\hline Mediana & \multicolumn{2}{|c|}{77,0} & \multicolumn{2}{|c|}{70,5} & \\
\hline Média & \multicolumn{2}{|c|}{75,5} & \multicolumn{2}{|c|}{71,0} & 0,448 \\
\hline Desvio Padrão & \multicolumn{2}{|c|}{10,0} & \multicolumn{2}{|c|}{15,8} & \\
\hline
\end{tabular}

*Qui-quadrado de Pearson ou Exato de Fisher

A única variável que se associou ao risco de aspiração foi trânsito oral, sendo que todos os pacientes sem disfagia tiveram trânsito oral adequado e entre os pacientes com disfagia, metade tiveram trânsito oral alterado (Tabela 5).
Em relação às associações entre a gravidade da disfagia (leve e moderada) e as características sociodemográficas, clínicas, do sistema estomatognático e da deglutição dos pacientes com DPOC, nenhuma foi associada ao risco de aspiração. 
Tabela 5. Associação entre risco de aspiração e variáveis do sistema estomatognático e deglutição $(\mathrm{N}=27)$

\begin{tabular}{|c|c|c|c|c|c|}
\hline \multirow{3}{*}{\begin{tabular}{|l} 
Características \\
Higienização oral
\end{tabular}} & \multicolumn{4}{|c|}{ Aspiração } & \multirow{3}{*}{ Valor-p* } \\
\hline & \multicolumn{2}{|c|}{ Ausente } & \multicolumn{2}{|c|}{ Presente } & \\
\hline & $\mathbf{N}$ & $\%$ & $\mathbf{N}$ & $\%$ & \\
\hline Adequada & 15 & 65,2 & 3 & 75,0 & \\
\hline Inadequada & 8 & 34,8 & 1 & 25,0 & 0,593 \\
\hline Total & 23 & 100,0 & 4 & 100,0 & \\
\hline Vedamento Labial & $\mathbf{N}$ & $\%$ & $\mathbf{N}$ & $\%$ & \\
\hline Adequado & 21 & 91,3 & 3 & 75,0 & \\
\hline Inadequado & 2 & 8,7 & 1 & 25,0 & 0,395 \\
\hline Total & 23 & 100,0 & 4 & 100,0 & \\
\hline Movimentação de Língua & $\mathbf{N}$ & $\%$ & $\mathbf{N}$ & $\%$ & \\
\hline Adequada & 21 & 91,3 & 3 & 75,0 & \\
\hline Alterada & 2 & 8,7 & 1 & 25,0 & 0,092 \\
\hline Total & 23 & 100,0 & 4 & 100,0 & \\
\hline Força de Língua & $\mathbf{N}$ & $\%$ & $\mathbf{N}$ & $\%$ & \\
\hline Adequada & 21 & 91,3 & 2 & 50,0 & \\
\hline Alterada & 2 & 8,7 & 2 & 50,0 & 0,092 \\
\hline Total & 23 & 100,0 & 4 & 100,0 & \\
\hline Coordenação de Língua & $\mathbf{N}$ & $\%$ & $\mathbf{N}$ & $\%$ & \\
\hline Adequada & 19 & 82,6 & 2 & 50,0 & \\
\hline Alterada & 4 & 17,4 & 2 & 50,0 & 0,204 \\
\hline Total & 23 & 100,0 & 4 & 100,0 & \\
\hline Reflexo de Tosse & $\mathbf{N}$ & $\%$ & $\mathbf{N}$ & $\%$ & \\
\hline Adequado & 22 & 95,7 & 4 & 100,0 & \\
\hline Alterado & 1 & 4,3 & 0 & 0,0 & 0,852 \\
\hline Total & 23 & 100,0 & 4 & 100,0 & \\
\hline Deglutição Salivar & $\mathbf{N}$ & $\%$ & $\mathbf{N}$ & $\%$ & \\
\hline Adequada & 20 & 87,0 & 3 & 75,0 & \\
\hline Alterada & 3 & 13,0 & 1 & 25,0 & 0,495 \\
\hline Total & 23 & 100,0 & 4 & 100,0 & \\
\hline Preparação Oral & $\mathbf{N}$ & $\%$ & $\mathbf{N}$ & $\%$ & \\
\hline Adequada & 22 & 95,7 & 3 & 75,0 & \\
\hline Alterada & 1 & 4,3 & 1 & 25,0 & 0,279 \\
\hline Total & 17 & 100,0 & 10 & 100,0 & \\
\hline Clareamento do Bolo & $\mathbf{N}$ & $\%$ & $\mathbf{N}$ & $\%$ & \\
\hline Adequado & 21 & 91,3 & 3 & 75,0 & \\
\hline Alterado & 2 & 8,7 & 1 & 25,0 & 0,395 \\
\hline Total & 23 & 100,0 & 4 & 100,0 & \\
\hline Trânsito Oral & $\mathbf{N}$ & $\%$ & $\mathbf{N}$ & $\%$ & \\
\hline Adequado & 23 & 100,0 & 2 & 50,0 & \\
\hline Alterado & 0 & 0,0 & 2 & 50,0 & 0,017 \\
\hline Total & 23 & 100,0 & 4 & 100,0 & \\
\hline Fase Faríngea & $\mathbf{N}$ & $\%$ & $\mathbf{N}$ & $\%$ & \\
\hline Adequada & 20 & 87,0 & 2 & 50,0 & \\
\hline Alterada & 3 & 13,0 & 2 & 50,0 & 0,144 \\
\hline Total & 23 & 100,0 & 4 & 100,0 & \\
\hline Resposta Faríngea & $\mathbf{N}$ & $\%$ & $\mathbf{N}$ & $\%$ & \\
\hline Adequada & 23 & 100,0 & 2 & 50,0 & \\
\hline Alterada & 0 & 0,0 & 2 & 50,0 & 0,128 \\
\hline Total & 23 & 100,0 & 4 & 100,0 & \\
\hline Frequência Respiratória & $\mathbf{N}$ & $\%$ & $\mathbf{N}$ & $\%$ & \\
\hline Adequada & 3 & 13,0 & 1 & 25,0 & \\
\hline Alterada & 20 & 87,0 & 3 & 75,0 & 0,495 \\
\hline Total & 23 & 100,0 & 4 & 100,0 & \\
\hline
\end{tabular}

* Teste Exato de Fisher 


\section{Discussão}

Os dados deste estudo demonstraram que pacientes com DPOC apresentaram disfagia e riscos de aspiração. Em um estudo, os autores sugeriram que apesar dos pacientes com DPOC não apresentarem queixas relativas à deglutição, apresentam riscos importantes para aspiração, uma vez que possuem alterações no padrão respiratório, o que pode alterar a coordenação entre a respiração e a deglutição, fundamental para a proteção da via aérea inferior ${ }^{17}$. O padrão inspiratório com a abertura das pregas vocais pode facilitar a entrada de alimentos e saliva na laringe durante ou após a deglutição, aumentando o risco de aspiração ${ }^{18}$

$\mathrm{Na}$ escala funcional de alimentação (FOIS), $44,5 \%$ dos pacientes apresentaram FOIS 1 após a avaliação da Fonoaudiologia, embora a gravidade da disfagia tenha sido considerada leve na maioria dos participantes, a via alternativa exclusiva de alimentação foi estabelecida logo após a avaliação fonoaudiológica devido às condições respiratórias desses pacientes internados por complicações da DPOC, indicando uma conduta fonoaudiológica mais cautelosa sobre a via de alimentação. Vale ressaltar que, a aspiração laríngea pode ser uma possível causa das exacerbações da DPOC ${ }^{19}$.

Um dos pacientes não-disfágicos foi classificado como FOIS 1 (nada por via oral), porque ele apresentou comprometimento no estado de alerta (flutuação do nível de consciência e cooperação distração por outros estímulos), sendo os demais itens do MASA adequados. Como o MASA é um protocolo de escores, esse paciente não atingiu pontos suficientes para ser classificado como disfágico.

Durante a fase oral, ocorre a preparação do alimento na cavidade oral por meio da mastigação e a transformação em um bolo alimentar coeso ${ }^{20}$. Dificuldades nessa fase interferem na qualidade e segurança da alimentação, sendo de fundamental importância, a cooperação do paciente ${ }^{21}$. Neste estudo a cooperação do paciente teve associação com presença de disfagia, o que corrobora com a literatura.

A fase faríngea e a resposta faríngea foram as alterações encontradas na população estudada, seguida do trânsito oral comprometido, no qual teve associação com o risco de aspiração. Pacientes com DPOC apresentam sintomas de disfagia relacionados à fase faríngea da deglutição, no que se refere ao mecanismo de proteção das vias aéreas ${ }^{8}$ Outros estudos verificaram tempo de trânsito oral prolongado $^{22}$, elevação laríngea reduzida durante a deglutição e alteração do músculo cricofaríngeo ${ }^{9,23}$. O controle motor oral ruim pode prejudicar a ejeção do bolo alimentar e assim ocorrer aspiração antes da deglutição, desta forma é imprescindível a avaliação minuciosa de todas as fases da deglutição nos pacientes com DPOC.

Embora a idade pudesse ter sido um fator que tivesse contribuído para a chance de se ter disfagia ou risco de aspiração, não foi observado associação na população estudada, uma vez que a média de idade foi 74 anos. Do ponto de vista anatômico e funcional, com o envelhecimento ocorrem redução da mobilidade da caixa torácica, da elasticidade pulmonar e diminuição dos valores da pressão inspiratória e expiratória máximas ${ }^{24}$. Além do comprometimento respiratório, a população idosa, por apresentar envelhecimento no mecanismo da deglutição, exprime maior risco para a ocorrência da disfagia, pela redução da sensibilidade orofacial, diminuição dos movimentos orais, perdas dentárias e pela utilização de próteses ${ }^{25}$.

Em relação às comorbidades, neste estudo, houve maior ocorrência de pneumonia e doenças metabólicas. Pacientes com DPOC apresentam infecções frequentes do trato respiratório inferior ${ }^{19}$ devido às alterações de deglutição ${ }^{23}$. Um estudo verificou presença de penetração e aspiração laríngea por meio da videofluoroscopia nos pacientes com DPOC $^{26}$. Dois estudos avaliaram a coordenação entre a deglutição e a respiração por meio da pletismografia e verificaram maior tempo de apneia e ocorrência da deglutição na fase inspiratória da respiração, o que torna favorável a aspiração ${ }^{27,28}$.

Apesar dos resultados indicarem associações entre a disfagia e o DPOC, o tamanho da amostra reduzido não permitiu análise estatística robusta. Além disso, não foi possível verificar a correlação entre o transtorno de deglutição e o grau de comprometimento do DPOC, pelo fato do hospital em que a coleta foi realizada, não ser referência em comorbidades respiratórias, assim o estadiamento do DPOC por meio da escala GOLD foi pouco frequente entre os participantes, inviabilizando a análise.

Todavia, os resultados encontrados indicaram presença de disfagia e risco de aspiração em pacientes internados com DPOC, sugerindo a necessidade de investigação da dinâmica da deglutição durante a hospitalização, inclusive com exames objetivos como a videofluoroscopia ou videoendoscopia da deglutição. Toda a equipe envolvida no tratamento 
desses pacientes deve estar atenta à possibilidade de ocorrência de disfagia, o que pode aumentar o risco de complicações durante a internação, sendo que a prevenção de broncoaspiração é um dos objetivos preconizados na segurança do paciente hospitalizado.

\section{Conclusão}

A disfagia esteve presente em $41,7 \%$ dos pacientes com DPOC que foram avaliados pela equipe de Fonoaudiologia, sendo a gravidade leve mais prevalente, e o risco de aspiração foi de 19\%. Pacientes pouco cooperativos e com tempo de trânsito oral aumentado foram as variáveis associadas à presença de disfagia e ao risco de aspiração, respectivamente.

Os resultados deste estudo sugerem que a investigação da habilidade de deglutição deve ser realizada de forma rotineira em todos os pacientes com DPOC hospitalizados, a fim de minimizar complicações clínicas relacionadas à disfagia.

\section{Referências bibliográficas}

1. Celli BR, MacNee W, Agusti A, Anzueto A, Berg B, Buist AS, et al. American Thoracic Society. Standards for the diagnosis and treatment of patients with COPD. Eur. respir. j. 2004; 23(6): 933-44.

2. Fabbri L, Pauwels RA, Hurd SS. Global Strategy for the Diagnosis, Management, and Prevention of Chronic Obstructive Pulmonary Disease: Gold executive summary updated 2003. Am J Respir Crit Care Med. 2004; 1: 105-41.

3. Oliveira JA, Jaraim JR, Rufino R. I. Consenso Brasileiro de Doença Pulmonar Obstrutiva Crônica. J. bras. pneumol. 2000; 26 (1): s4-s44.

4. National Institutes of Healt, Morbitiy\& Mortality. National Heart, Lungc and Blood Institute: chart book on cardiovascular, lung and blood diseases. http://www.nhlbi.nih.gov/resources/ docs/cht-book.htm. Updated April 2012. Accessed ago 25, 2015.

5. Rabahi MF. Epidemiology of COPD: facing challenges. Pulmão RJ. 2013; 22(2): 4-8.

6. Menezes AM, Jardim JR, Perez-Padilla R, Camelier A, Rosa F, Nascimento O, et al. Prevalence of chronic obstructive pulmonary disease and associated factors: the Platino Study in Sao Paulo Brazil. Cad. saúde pública. 2005; 21(5): 1565-73.

7. Marchiori RC, Susin CF, Lago LD, Felice CD, Silva DB, Severo MD. Diagnóstico e tratamento da DPOC exacerbada na emergência. Rev da AMRIGS. 2010; 54 (2): 214-23.

8. Chaves RD, Carvalho CRF, Cukier A, Stelmach Rl, Andrade CRF. Symptoms of dysphagia in patients with COPD. J Bras Pneumol. 2011; 37(2): 176-83.

9. O'Kane L, Groher M. Oropharyngeal dysphagia in patients with chronic obstructive pulmonary disease: a systematic review. Rev. CEFAC. 2009; 11(3): 449-506.
10. Clayton NA, Carnaby-Mann GD, Peters MJ, Ing AJ. The effect of chronic obstructive pulmonary disease on laryngopharyngeal sensitivity. Ear nose throat j. 2012; 91(9): 370-4.

11. Singh B. Impaired swallow in COPD. Respirol. 2011; 16(2): $185-6$

12. Chaves RD, Sassi FC, Mangilli LD, Jayanthi SK, Cukier A, Zilberstein B. Swallowing transit times and valleculae residue in stable chronic obstructive pulmonary disease.BMC pulm. med. 2014; 62(14): 2-9.

13. Silva RG. A eficácia da reabilitação em disfagia orofaríngea. Pró-Fono. 2007; 19(1): 123-30. Furkim AM, Sacco ABF. Efficacy of speech therapy in neurogenic dysphagia using functional oral intake scale (FOIS) as a parameter. Rev CEFAC. 2008; 10(4): 503-12.

14. Mann G. The Mann Assessment of Swallowing Ability: MASA. Philadelphia; Delmar Thompson Learning. 2002.

15. Crary MA, Mann GD, Groher ME. Initial psychometric assessment of a functional oral intake scale for dysphagia in stroke patients. Arch Phys Med Rehab. 2005; 86 (8): 1516-20.

16. Drozdz DRC, Costa CC, Jesus PRO, Trindade MS, Weiss G, Neto ABM, et al. Análise da fase faríngea da deglutição em portadores de tosse crônica. Int. Arch. Otorhinolaryngol. 2012; 16(4): $502-8$.

17. Martin-Harris B, Brodsky MB, Price CC, Michel Y, Walters B. Temportal coordination of pharyngeal and laryngeal dynamics with breathing during swallowing: single liquid swallows. J Appl Physiol. 2003; 94(5): 1735-43.

18. Kobayashi S, Kubo H, Yanai M. Impairment of the swallowing reflex in exacerbations of COPD. Thorax. 2007; 62(11) 1017.

19. Marchesan IQ. Deglutição-Normalidade. In: Furkim AM, Santini CS. Disfagias Orofaríngeas. Pró-Fono, 1999:3-18.

20. Mendell DA, Logemann JA. Temporal Sequence of Swallow events During the Oropharyngeal Swallow. J Speech Lang Hear Res. 2007; 50 (5): 1256-71.

21. Mokhlesi B, Logemann JA, Rademaker AW, Stangl CA, Corbridge TC. Oropharyngeal deglutition in stable COPD. Chest. 2002; 121(2): 361-9

22. Rabe KF, Beghé B, Luppi F, Fabbri LM. Update in Chronic Obstructive Pulmonary Disease 2006. Am J Respir Crit Care Med 2007; 175: 1222-32.

23. Francisco MSB, Donasilio MR, Barros MBA, César CLG, Carandina L, Goldbaum M. Fatores associados à doença pulmonar em idosos. Rev Saúde Pública. 2006; 40(3): 428-35.

24. Tanure CMC, Barboza JP, Amaral JP, Motta AR. A deglutição no processo normal de envelhecimento. Rev CEFAC. 2005. 7(2): 171-7.

25. Good-Fratturelli MD, Curlee RF, Holle JL. Prevalence and nature of dysphagia in VA patients with COPD referred for videofluoroscopic swallow examination. J Commun Disord. 2000; 33(2): 93-110.

26. Gross RD, Atwood Jr CW, Ross SB, Olszewski JW, Eichhorn KA. The Coordination of Breathing and Swallowing in Chronic Obstructive Pulmonary Disease. Am J Respir Crit Care Ned. 2009; 179(7): 559-65.

27. Shaker R, Li Q, Ren J, Townsend WF, Dodds WJ, Martin BJ, et al. Coordination of deglutition and phases of respiration: effect of aging, tachypnea, bolus volume, and chronic obstructive pulmonary disease. Am J Physiol. 1992; 263: G750-5. 